\title{
Orthokeratology for the Prevention of Myopic Progression in Children
}

\author{
An Expert Interview with Deborah $\mathrm{K}$ VanderVeen \\ Department of Ophthalmology, Boston Children's Hospital, Boston, MA, USA
}

DOl: https://doi.org/10.17925/USOR.2020.13.1.12

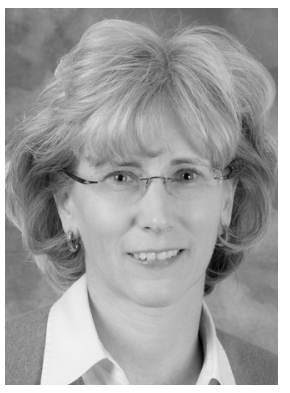

\begin{abstract}
Deborah K Vanderveen
Deborah K VanderVeen, MD, holds an Endowed Clinical Chair in the Department of Ophthalmology at Boston Children's Hospital, and is an Associate Professor in the Department of Ophthalmology at Harvard Medical School. After residency training at Boston Medical Center, she completed a fellowship in pediatric ophthalmology and strabismus. She has special clinical and research interests in the management of pediatric cataract and retinopathy of prematurity, and has been the site principal investigator for several National Institutes of Health-sponsored clinical trials. Dr VanderVeen is an active member of the New England Ophthalmological Society, the American Association of Pediatric Ophthalmology and Strabismus (AAPOS), the American Academy of Ophthalmology (AAO), and the American Society of Cataract and Refractive Surgery, and has received the Senior Honor Award from both the AAO and the AAPOS. She has published numerous peer-reviewed papers and is an Associate Editor for the Journal of AAPOS. She performed a review of the literature on use of orthokeratology for the prevention of myopia progression in children as a member of the AAO's Ophthalmic Technology Assessment Committee, which was published in 2019.
\end{abstract}

\section{Keywords}

Orthokeratology, ortho-k lenses, myopia, pediatric ophthalmology

Disclosure: Deborah K Vanderveen has no financial or non-financial relationships or activities to declare in relation to this article.

Review Process: This is an expert interview and as such has not undergone the journal's standard peer review process.

Compliance with Ethics: This article is an expert interview and does not report on new clinical data, or any studies with human or animal subjects performed by the author.

Authorship: The named author meets the International Committee of Medical Journal Editors (ICMJE) criteria for authorship of this manuscript, takes responsibility for the integrity of the work as a whole, and has given final approval for the version to be published.

Access: This article is freely accessible at tOuchOPHTHALMOLOGY.COM (C) Touch Medical Media 2020

Received: November 18, 2019

Published Online: June 9, 2020

Citation: US Ophthalmic Review. 2020;13(1):12-3

Corresponding Author: Deborah K VanderVeen Department of Ophthalmology, Boston Children's Hospital, 300 Longwood Avenue

Boston, MA 02115, USA.

E: deborah.vanderveen@childrens.harvard.edu

Support: No funding was received in

the publication of this article. n this Expert Interview, we speak to Dr Deborah $\mathrm{K}$ VanderVeen about the rationale for the use of orthokeratology for the prevention of myopic progression in children, and the conclusion of the recent American Academy of Ophthalmology (AAO) report on this subject. She also discusses which children it would likely benefit and those in whom it is contraindicated, and the major safety implications

\section{Q: What is the rationale for the use of orthokeratology for the prevention of myopic progression in children?}

Overnight orthokeratology (ortho-k) lenses are worn to flatten the central cornea, which results in peripheral hyperopic defocus on the retina. This is thought to slow the elongation of the eye (slow axial growth). Less elongation of the eye results in less progression of the myopia.

\section{Q: What was the conclusion of the recent AAO report on this subject?}

The Ophthalmic Technology Assessment Committee studies interventions to look at evidence for efficacy, but the intent is not to endorse or negate the effects of technologies. The clinical studies reviewed overall show a statistically significant slowing of axial length growth in the groups of children who wore ortho-k lenses, compared with control groups of children who used spectacle or daytime soft contact lenses for the correction of myopia. ${ }^{1}$ Most of the patients were between 8 and 16 years of age, and most were of Asian ethnicity. Concerns about the studies were small numbers of patients, high drop-out rates, and small and variable clinical effects. In addition, there are also concerns about the cost of the treatment and safety, since any contact lens wear carries with it the risk of microbial keratitis, which is potentially blinding. ${ }^{1}$

\section{Q: Which children are most likely to benefit from this treatment, and in whom is it contraindicated?}

The children in the study were mostly of Asian ethnicity, so it is unknown whether there is genetic influence with regard to treatment response. ${ }^{1}$ There is a suggestion that younger children can benefit more, and that combining ortho-k treatment with atropine (low-dose) may have a synergistic effect. ${ }^{1}$ 


\section{Q: What are the major safety concerns associated with this procedure, and how can the risks be minimized?}

The major safety concern is microbial keratitis. This can be minimized by proper fitting of the lenses, with careful oversight by an optometrist certified in ortho-k treatment, regular follow-up, and good contact lens care. There is still a risk of infection with contact lenses, and this may be higher with overnight wear of contact lenses. In contrast, there is no such risk with comparable treatments, such as spectacle wear with low-dose atropine. ${ }^{1} \square$

1. VanderVeen DK, Kraker RT, Pineles SL, et al. Use of orthokeratology for the prevention of myopic progression in children. Ophthalmology. 2019;126:623-36.

\section{toUChPANEL DISCUSSION}

\section{HOW DO WE IMPROVE THE STANDARD OF CARE IN CATARACT SURGERY?}

\section{Pouch \\ OPHTHALMOLOGY}

